\title{
Stable Grinding Micro-wall of Flexible Joints with Controllable Force
}

\author{
Da $\mathrm{Qu}^{1}$, Fei Ding ${ }^{1}$ and Bo Wang ${ }^{1, a}$ \\ ${ }^{1}$ CPE, Harbin Institute of Technology, Harbin, 150001, P. R. China
}

\begin{abstract}
The micro-wall between two adjacent pores on the flexible joint is the core structure affecting the performance of gyro. The thickness of the micro-wall is only about $30 \mu \mathrm{m}$ and decreases to $20 \mu \mathrm{m}$ after being machined. It is a low-stiffness structure easily affected by grinding force. Larger force can break the micro-wall. Hence, appropriate grinding force must be controlled to make balance between machining efficiency and surface quality. Besides, different thickness before being machined affects the machining stability as well. The present paper firstly introduces the developed machine tool used for grinding the micro-walled. With this machine tool, removal of the process can be measured as well. Detailed measuring theory and grinding processes are presented. The force can be adjusted and monitored in the microgrinding process. To achieve stable removal, the related thickness is analysed by experiments. With this machine tool, the process of machining-measuring micro-wall is efficient.
\end{abstract}

\section{Introduction}

The flexible joint is the core part of dynamically tuned gyro (DTG) which is widely used in avigraph. Varying structures and principles of gyro started to appear in 1942. There into, DTG is highly required in the manufacturing field of aircrafts and missiles due to its small size, high precision and high reliability [1]. The working part of flexible joints, the micro-wall, is shown in figure 1.
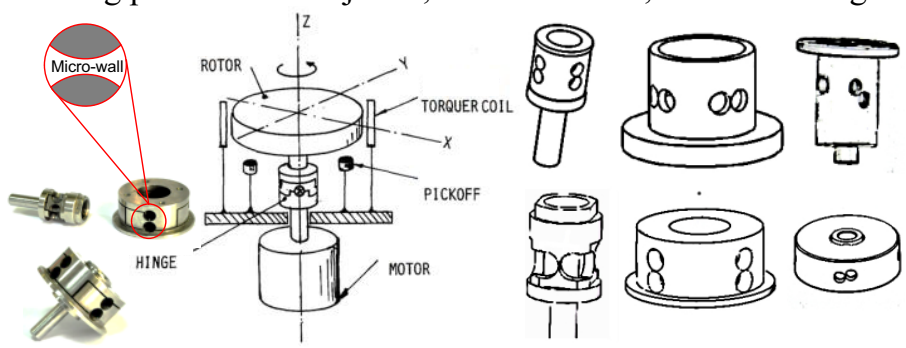

Figure 1. Components and operating principle of DTG

In service process of the flexible joint, the micro-wall is always broken firstly. Hence, it is very significant to enhance its dimensional accuracy and processed quality. As its importance, many researchers carried out relevant studies on micro machining. Wang et al. proposed a composite

\footnotetext{
${ }^{\mathrm{a}}$ Corresponding author : bradywang@hit.edu.cn
} 
processing technology which combines electrical discharge machining with ultrasonic vibrating grinding to process micro-pores [2]. A spiral polishing method applied in finishing the micro-pores is proposed by Yan et al. [3]. This method makes good processing accuracy but only can be used in processing a through hole. Ultrasonic assisted grinding technology was carried out by Peng et al.[4-6]. Their experiments show good performance on obtaining high quality surface. Yi et al.[7] developed a pen shaped controlling polishing device for polishing optical mold. Brinksmeier et al. [8,9] advanced thorough research of polishing microstructure array employing multiaxial finishing equipment which is suitable for studying removal mechanism. Guo et al. [10-12] and Suzuki et al. [13,14] developed a real-time force feedback controlling system to process ultra-precision optical surface. However, for stable removal, little researches were studied.

In this paper, a machine tool with two-dimensional force feedback is developed. With the twodirectional force feedback, it is easy to measurement and process the micro-wall. A series of experiments were carried out and the relationship between the thickness and removal amount is illustrated. Detailed processing theory and measuring method were presented as well.

\section{Setup}

Figure 2 shows the structure of the machine tool which is applied to grind the micro-pore. They consist of force sensor, moving components, and control system.

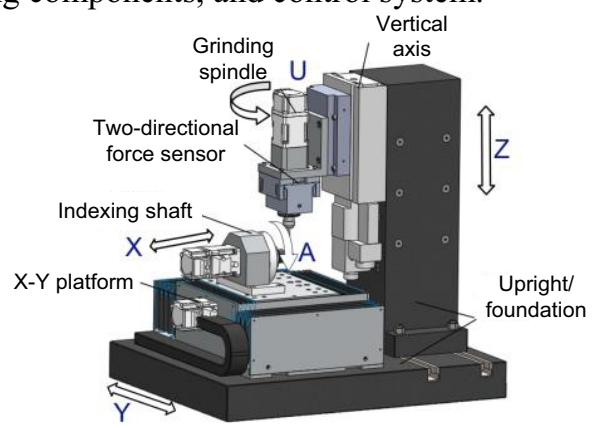

Figure 2. Structural distribution of the machine tool

Forece sensor: The force sensor was made of magnalium. Four strain gages with high sensitive coefficient were attached on each sensitive spot. They consist of a half bridge arms form of Wheatstone bridge. The force resolution of the sensor is $6 m N$ which occupies $0.06 \%$ of the whole measuring range after its compensation since the force sensor is anisotropy. The direction of the force can be measured by two directional force components as well.

Moving components: Five axes are used in the machine tool. X, Y and Z axis is used for linear movement. Axis $\mathrm{A}$ is applied to adjust the cocoaxiality between the micro-pore and $\mathrm{Z}$ axis. The directional moving accuracy reaches about $50 \mathrm{~nm}$. The repeated accuracy in $Z$ direction is $300 \mathrm{~nm}$. Axis $U$ makes the grinding bar rotate. The angular displacement resolution of axis $U$ is 1.24 second of arc. Rotation precision of axis $\mathrm{A}$ is less than $1 \mu \mathrm{m}$.

Control system: The hardware components of the control system are elaborated as figure $3 \mathrm{a}$. Dynamic strain meter and high-speed data acquisition system are employed. Moving control module is composed of motion control card, actuators, feedback grating, main power source and control power supply circuit.

Software: The software used for controlling the movement of each axes, data output and input, and monitoring the grinding process is shown in figure $3 \mathrm{~b}$. Functions of different areas in the software are pointed out. The display area shows the captured force amplitude and its direction. 

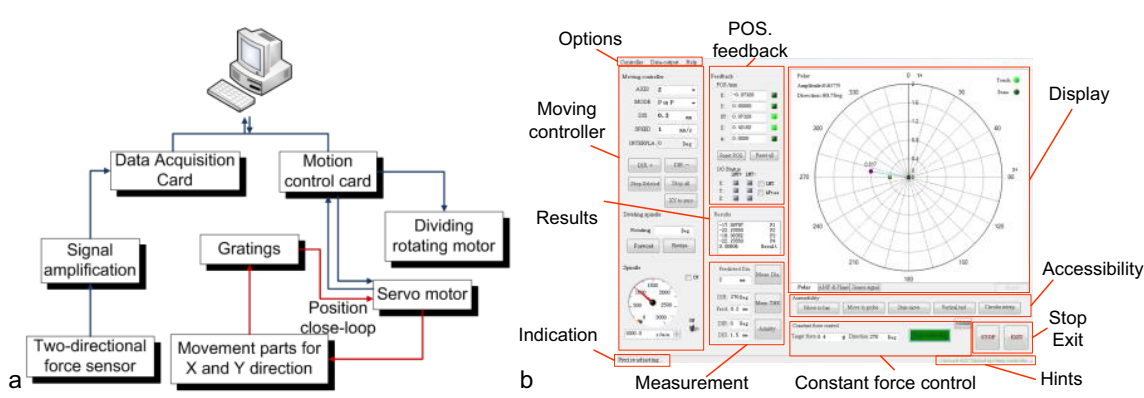

Figure 3. Schematic diagram: a. Components of control system b. The software and its functions

\section{Processing steps}

This part illustrates some key steps of micro-grinding flexible joints, including adjustement of coaxiality, choice of grinding bar and measurement of removal. All these steps affect the accuracy of measuring and machining.

\subsection{Adjustment of coaxiality}

Before micro grinding, coaxiality between the micro-pore and $\mathrm{Z}$ direction should be adjusted. The difference of the coaxiality is elaborated in figure 4.

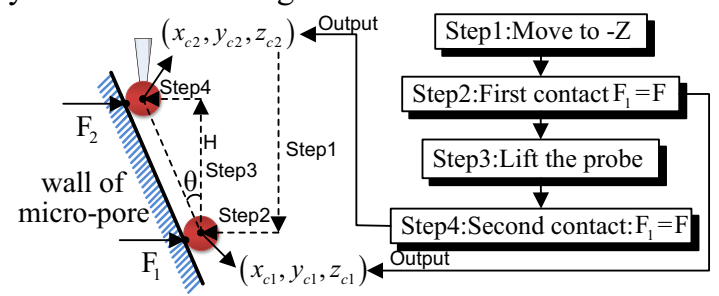

Figure 4. Flow diagram of coaxiality measurement

In the coaxiality measurement, the micro-wall is moved to make contact with the probe until $F_{1}=F$. The coordinate after being compensated is put out. Where the compensation of the deformation of probe and force can be done by measuring a fixed gauge block. $\left(x_{c 1}, y_{c 1}, z_{c 1}\right)$ and $\left(x_{c 2}, y_{c 2}, z_{c 2}\right)$ are the calculated coordinates in the first and second contact after compensation, respectively. $F_{1}$ is the reactive force on the probe from the wall in the first contact. $F$ is the contacting force which can be set to make the judgement of contact. $H$ is the height of the lifting distance which needs setting before the measurement. The coaxiality can be calculated as

$$
\theta=\arctan \frac{\sqrt{\left(x_{c 2}-x_{c 1}\right)^{2}+\left(y_{c 2}-y_{c 1}\right)^{2}}}{H}
$$

Rotating direction of axis A can be judged with sign symbol of $\theta$. The origin of coordinates can be set as any position in the moving range of axes.

\subsection{Selection of grinding bar}

In the grinding process, the material of the grinding tool significantly impacts the machined dimensional accuracy, efficiency and surface quality. The grinding paste is applied between the micro-wall and the grinding bar. Grinding bar made of cast iron is used in this experiment. Besides, 
the size of the grinding bar needs choosing since directional grinding generates unrounded profile which affects the performance of the micro-wall.
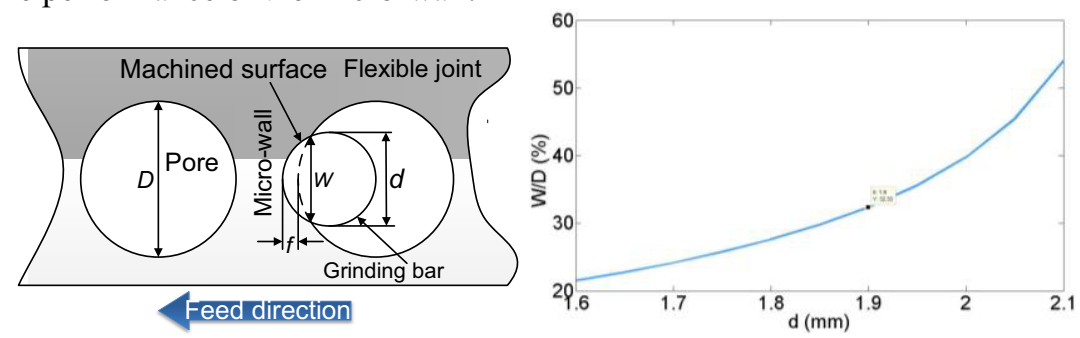

Figure 5. Schematic diagram of geometrical relationship in grinding process

The relationship between diameter of grinding bar and machined width is shown as figure 5 and is presented as

$$
D-d+2 f+\sqrt{d^{2}-W^{2}}-\sqrt{D^{2}-W^{2}}=0 \quad\left(2 f \leq \sqrt{D^{2}-W^{2}}+d-D\right)
$$

Where $\mathrm{D}$ denotes the diameter of pore, $\mathrm{d}$ denotes the diameter of grinding bar, $\mathrm{W}$ is the machined width and $f$ is the feed of grinding bar. In this experiment, $D$ is $2.2 \mathrm{~mm}$. $f$ is $0.01 \mathrm{~mm}$ and the appropriate $\mathrm{d}$ can be chosen from the relationship between W/D and $\mathrm{d}$. W/D should be larger where possible. However, the chosen $\mathrm{d}$ should be smaller than $\mathrm{D}$ since the grinding bar can insert the pore easily. Consider its balance, diameter of the grinding bar used in the experiment is $1.9 \mathrm{~mm}$ which makes $\mathrm{W} / \mathrm{D}$ reaches over $30 \%$.

\subsection{Measurement of removal}

The thickness measuring theory is elaborated in figure 6 . In step 2, move the platform to make the micro-wall contact with the probe and precise adjusting until the captured reactive force $F_{1}$ reaches the set contacting force $F$. Then, the contacing position coordinate $\left(x_{c 1}, y_{c 1}\right)$ calculated after deforming compensation of the sensor and probe is put out. After the first contact is fullfiled, repeating the first contact to fullfil a second contact with the other side of the micro-wall in the same depth $D_{c}$ with the first-time contact to obtain the contacting position coordinate $\left(x_{c 2}, y_{c 2}\right)$. Where $D$ is the depth from the upper surface of the wall. Then thickness $T_{w}$ can be calculated as

$$
T_{w}=\sqrt{\left(x_{c 1}-x_{c 2}\right)^{2}+\left(y_{c 1}-y_{c 2}\right)^{2}}-2 R_{p}
$$

Where $R_{p}$ denotes the radius of the probe head. The repeated accuracy of measuring thickness in $\mathrm{X}$ direction is $\pm 42 \mathrm{~nm}$.
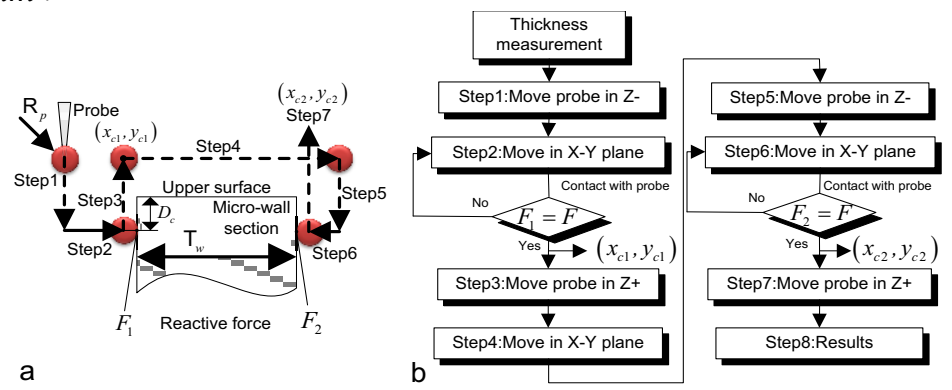

Figure 6. Thickness measuring theory

\section{Experimental results}




\subsection{Experimental setting}

In this experiment, the applied machine tool is shown in figure 7. Some setting details are shown in table 1. Diamond grinding paste with W2.5 was applied in this experiment. The material of flexible joint is a sort of high elastic alloy whose brand is 3J33A. In addition, the reciprocating movement of axis $\mathrm{Z}$ was taken in the experiment to complicate the machining track and decrease the surface roughness.

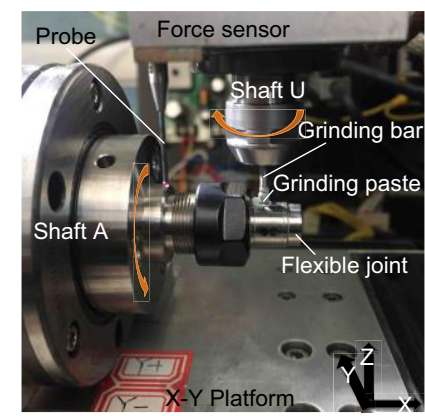

Figure 7. The machine tool used for micro grinding

Table 1. The set parameters in micro grinding process

\begin{tabular}{cccc}
\hline$F(\mathrm{~N})$ & $R_{p}(\mathrm{~mm})$ & $H(\mathrm{~mm})$ & $D_{c}(\mathrm{~mm})$ \\
\hline 0.1 & 0.5 & 1.5 & 1.5 \\
\hline
\end{tabular}

\subsection{Stably grinding}

In this grinding experiment, the used parameters are listed in table.2. The grinding time is $60 \mathrm{~s}$. The original thickness was measured before the micro-grinding was taken and the removal amount can be calculated by the difference between the original thickness and the thickness after machined. The relationship between the original thickness and the removal amount is shown in figure 8 . Three-times measurements were taken in a same grinding experiment.

Table 2 The set parameters in micro grinding process

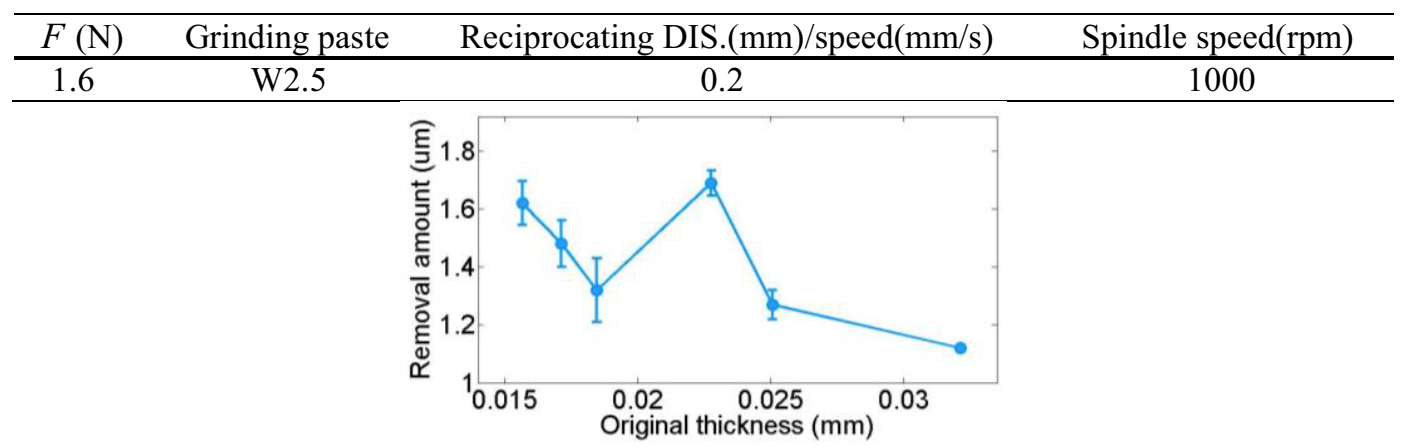

Figure 8. The relationship between the original thickness and removal amount

In figure 8, the removal amount decreases with the increase of original thickness. As the smaller thickness has larger elastic deformation when the same grinding force is applied, more grinding paste can flow between the grinding bar and the micro-wall. This causes the larger removal amount. Besides, the removal amount changes in range of $480 \mathrm{~nm}$ when the thickness changes in range of $4.5 \mathrm{um}$. 


\section{Conclusions}

The present developed machine tool performs well on stably grinding the internal micro-wall. With the force feedback, grinding efficiency can be enhanced with high-stability. In addition, appropriate grinding parameters can be chosen aiming at varying thickness. Some conclusions can be expounded as

(1) Appropriate grinding force can be chosen with the force feedback in case of breaking the micro-wall.

(2) Fast measurement of the micro-wall can be carried out without clamping again which takes clamping error. The coaxiality of the flexible joint can be measured and adjusted to decrease grinding error.

(3) The measured thickness has measuring error since when contacting with the probe, elastic deformation appears to decrease the measuring results of the thickness. The removal amount is affected by the viscosity of the grinding paste as well. This induces the experimental results.

(4) With the feedback of grinding force, more applications can be achieved. For further study, the grinding time can be taken as an index of removal amount under the condition of certain processing parameters.

\section{References}

1. M.B. Bafghi, A. Vahedi, J. Soleimani. Optimized Design of PM Torquer for Dynamically Tuned Gyroscope. IEEE. T. Ind. Appl. 48 (2012)

2. A.C. Wang, B.H. Yan, X.T. Li, F.Y. Huang. Use of micro ultrasonic vibration lapping to enhance the precision of microholes drilled by micro electro-discharge machining. Int. J. Mach. Tool. Manu. 42 (2002)

3. B.H. Yan, H.J. Tzeng, H.F. Yuan, Y.C. Lin, H.M. Chow. Finishing effects of spiral polishing method on micro lapping surface. Int. J. Mach. Tool. Manu. 47 (2007)

4. Y. Peng, Z. Liang, Y. Wu, Y. Guo, C. Wang. Characteristics of chip generation by vertical elliptic ultrasonic vibration-assisted grinding of brittle materials. Int. J. Adv. Manuf. Tech. 62 (2011)

5. Y. Peng, Z. Liang, Y. Wu, Y. Guo, C. Wang. Effect of vibration on surface and tool wear in ultrasonic vibration-assisted scratching of brittle materials. Int. J. Adv. Manuf. Tech. 59 (2011)

6. Y. Peng, Y.B. Wu, Z.Q. Liang, Y.B. Guo, X. Lin. An experimental study of ultrasonic vibrationassisted grinding of polysilicon using two-dimensional vertical workpiece vibration. Int. J. Adv. Manuf. Tech. 54 (2010)

7. A.Y. Yi, M. Hezlep, T. Pol. A computer controlled optical pin polishing machine. J. Mater. Process. Tech. 146 (2004)

8. E. Brinksmeier, O. Riemer, A Gessenharter. Finishing of structured surfaces by abrasive polishing. Precis. Eng. 30 (2006)

9. E. Brinksmeier, O. Riemer, A. Gessenharter, L. Autschbach. Polishing of Structured Molds. CIRP. Ann-Manuf. Techn. 53 (2004)

10. J. Guo, S-y. Morita, M. Hara, Y. Yamagata, T. Higuchi. Ultra-precision finishing of micro-aspheric mold using a magnetostrictive vibrating polisher. CIRP. Ann-Manuf. Techn. 61 (2012)

11. J. Guo, H. Suzuki, T. Higuchi. Development of micro polishing system using a magnetostrictive vibrating polisher. Precis. Eng. 37 (2013)

12. J. Guo, H. Suzuki, S-y. Morita, Y. Yamagata, T. Higuchi. A real-time polishing force control system for ultraprecision finishing of micro-optics. Precis. Eng. 37 (2013)

13. H. Suzuki, S. Hamada, T. Okino, M. Kondo, Y. Yamagata, et al. Ultraprecision finishing of microaspheric surface by ultrasonic two-axis vibration assisted polishing. CIRP. Ann-Manuf. Techn. 59 (2010)

14. H. Suzuki, T. Moriwaki, T. Okino, Y. Ando. Development of Ultrasonic Vibration Assisted Polishing Machine for Micro Aspheric Die and Mold. CIRP. Ann-Manuf. Techn. 55 (2006) 